\title{
Electropolishing of stainless steels in a choline chloride based ionic liquid: an electrochemical study with surface characterisation using SEM and atomic force microscopy
}

\author{
Andrew P. Abbott, ${ }^{a}$ Glen Capper, ${ }^{a}$ Katy J. McKenzie, ${ }^{a}$ Andrew Glidle ${ }^{b}$ and \\ Karl S. Ryder* $a$
}

Received 31st May 2006, Accepted 14th July 2006

First published as an Advance Article on the web 3rd August 2006

DOI: $10.1039 / \mathrm{b607763n}$

\begin{abstract}
We have studied the anodic dissolution (electropolishing) of various stainless steel alloys in an ionic liquid comprising a 2:1 stoichiometric mix of ethylene glycol (EG) and choline chloride. We have used a combination of electrochemical and spectroscopic methods together with in situ liquid probe microscopy. We discuss the role and influence of the surface oxide passivation layer, characterized here by X-ray photoelectron spectroscopy (XPS) and linear sweep voltammetry, on the polishing process. We address the question of dealloying during the polish in order to contribute to our understanding of the viability of the ionic liquid as a replacement industrial electropolishing medium; the current commercial process uses a corrosive mixture of phosphoric and sulfuric acids. Also, we present data from ex situ and in situ liquid AFM studies giving both a qualitative and quantitative insight into the nature and scale of morphological changes at the steel surface during the polishing process.
\end{abstract}

\section{Introduction}

The process of electropolishing is one of controlled dissolution of a metal surface to bring about a reduction in surface roughness and hence an increase in optical reflectivity. The earliest systematic study of electropolishing led to a patent in 1930. ${ }^{1}$ The majority of research in this area has been carried out on stainless steel although metals such as copper, nickel and titanium have also been studied. ${ }^{2-4}$ The present stainless steel electropolishing process is performed worldwide on a commercial scale and is based on mixtures of concentrated phosphoric and sulfuric acids. The polishing process is thought to involve the formation of a viscous layer at the metal surface and as a result many processes employ viscosity improvers such as glycerol. The practical aspects of electropolishing have been reviewed recently ${ }^{5}$ following on from a description of the more fundamental aspects. ${ }^{6}$ While electropolishing is an extremely successful process there are major limitations and practical issues associated with the technology; most notably that the solution used is highly corrosive and toxic, extensive gas evolution (with associated low current efficiency) also occurs during the process.

During the last twenty years ionic liquids have found application in a wide variety of electrochemical and synthetic processes. $^{7-10}$ Most of the academic studies on ionic liquids have concentrated on imidazolium and pyridinium cations, however their high cost and sensitivity to water has made them of little practical use for large-scale applications such as metal finishing. Eutectic mixtures of zinc, tin and iron halides, in

\footnotetext{
${ }^{a}$ Chemistry Department, University of Leicester, Leicester, UK LE1 7RH.E-mail:ksr7@le.ac.uk; Fax: +44(0)1162523789

${ }^{b}$ Department of Electronics and Electrical Engineering, University of Glasgow, Glasgow, UK G12 8LT
}

combination with one of a variety of quaternary ammonium salts, can be easily formulated and used for metal finishing. ${ }^{11-14}$ These ionic liquids are air and moisture stable, the components are widely available, relatively benign and inexpensive allowing their usage in large-scale applications such as metal deposition. For example zinc and zinc alloys can be electrodeposited with high current efficiency from these ionic liquids. ${ }^{13,14}$ This technology has been extended to other metals by using hydrated metal salts. ${ }^{15,16}$ For example, mixtures of $\mathrm{CrCl}_{3} \cdot 6 \mathrm{H}_{2} \mathrm{O}$ with choline chloride form a liquid that can be used for the efficient electrodeposition of chromium.

The principle behind the formation of an ionic liquid by complexation of the halide anion has been extended by mixing quaternary ammonium salts with compounds that form hydrogen bonds. This has been demonstrated with a range of hydrogen bond donors including amides such as urea ${ }^{17}$ and carboxylic acids such as oxalic acid. ${ }^{18}$ We have previously shown ${ }^{19}$ that ethylene glycol can also be used as the hydrogen bond donor, and that mixtures of ethylene glycol with choline chloride can be used for the electropolishing of stainless steel. $^{20}$ This process has three main advantages over the commercial alternative: (i) high current efficiencies are obtained; (ii) gas evolution at the anode/solution interface during polishing is negligible; and (iii) the liquid used is comparatively benign and non-corrosive compared to the current aqueous acid solutions. In a previous study we have presented a.c. impedance, linear sweep voltammetry (LSV) and chronoamperometric data in an attempt to investigate the mechanism of electropolishing in these glycol mixtures. ${ }^{20}$ An interpretation of the data from that study, focussed specifically on 316 grade stainless steel, suggested that the formation and dissolution of a metal oxide passivation layer at the metal-liquid interface is a key feature of the metal dissolution mechanism 
and one that ultimately determines the quality of the optical finish (i.e. the surface roughness). It is clear that the differences in bulk composition for different grades of steel will influence the nature and stability of this oxide film, however, we have sought to develop an alternative electropolishing process that can encompass a range of different alloys. Consequently in the current investigation we studied the electropolishing process on a variety of stainless steel grades (SS410, 302, 304, 316 and 347). Also we report our findings on the variation of surface and sub-surface composition together with morphological changes that accompany the electropolishing process. We have used a combination of electrochemical techniques e.g. LSV, and surface probes including scanning electron microscopy (SEM), atomic force microscopy (AFM) and X-ray photoelectron spectroscopy (XPS).

\section{Experimental}

Choline chloride (ChCl) (Aldrich 99\%) was recrystallised from absolute ethanol, filtered and dried under vacuum. Ethylene glycol (EG) (Aldrich 99+\%) was used as received. The mixture was formed by stirring the two components $(1 \mathrm{ChCl}$ : 2 EG) at $75{ }^{\circ} \mathrm{C}$ until a homogeneous colourless liquid was formed. Voltammetry was carried out using an Autolab PGSTAT12 potentiostat controlled using GPES software. A three-electrode system consisting of a stainless steel (SS410, $302,304,316$ or 347$)$ disc working electrode ( $0.5 \mathrm{~mm}$ radius), sealed in glass, a platinum counter electrode and a silver wire reference electrode were used. The working electrode was abraded with 150 grit glass paper, rinsed and dried prior to all measurements to ensure reproducible voltammetric behaviour. Electrochemical measurements were performed at $20^{\circ} \mathrm{C}$ and a scan rate of $20 \mathrm{mV} \mathrm{s}^{-1}$ was used in voltammetric experiments except where stated.

Surface analysis was carried out using scanning electron microscopy (SEM) (Philips XL30 ESEM). Samples of the different grades of steel were examined using EDX prior to treatment in order to confirm compositional compliance with the AISI grading defined in Table 1. XPS data were acquired using the Scienta ESCA300 instrument at the NCESS facility, Daresbury laboratory. Samples were subjected to Ar ion etching by filling the sample preparation chamber of the Scienta instrument with Ar gas to a pressure of approximately $2 \times 10^{-6}$ Torr. The sample was then exposed to the Ar ion beam for a time period of typically between 1-2 min using an accelerator voltage of $5 \mathrm{kV}$ resulting in a beam current of $200 \mu \mathrm{A}$. The AFM images were obtained using a Digital Instruments Nanoscope IV Dimension 300 (Veeco) with a $100 \mu \mathrm{m}$ scanning head and run using both contact and tapping (resonant) modes. Images were acquired both in air and also in the liquid environment. The latter procedure was performed using the Veeco liquid attachment with the corresponding probes.

\section{Results and discussion}

Stainless steels are alloys composed mostly of $\mathrm{Fe}$ in combination with $\mathrm{Ni}$ and $\mathrm{Cr}$ often with small quantities of other metals such as $\mathrm{Mn}, \mathrm{Mo}, \mathrm{Nb}$, and Ta together with trace amounts of $\mathrm{C}$, $\mathrm{Si}, \mathrm{P}$ and $\mathrm{S}$. Compositional ranges for the standard grades (AISI) of stainless steels studied here are summarised in Table 1. The detailed chemistries of the different standard grades are well known, with the minor components playing important roles in the metallurgical properties of the steels. For example the extent to which $\mathrm{C}$ is present as a solid solution in $\mathrm{Fe}$ is quite different for 400 series steels (martensitic, low Ni) than that for 300 (austenitic) series materials. This has a strong influence on the hardness and tempering of the steel. Addition of other metals such as $\mathrm{Mo}, \mathrm{Nb}$ and Ta have stabilising effects, for example $\mathrm{Nb}$ and $\mathrm{Ta}$ are added to ss347, Table 1, in stoichiometric excess, with respect to $\mathrm{C}$, in order to prevent formation of $\mathrm{Cr}$ carbides. All of these subtleties determine the bulk phase structure of the steel and contribute to the surface properties such as corrosion resistance. The $\mathrm{Cr}$ content, in particular, is critical to the corrosion resistance of the steel since it encourages the formation of a mixed oxide film on the surface. Our previous study on the in situ electrochemical a.c. impedance during electropolishing of 316 grade stainless steel in an ethylene glycol (EG)/choline chloride $(\mathrm{ChCl})$ mixtures has suggested that the properties of this metal oxide film at the surface, rather than the bulk phase structure of the steel, has the most significant influence on the quality of the optical finish obtained. ${ }^{19,20}$ Consequently, this study has primarily focussed not on the individual roles of the minor components of the bulk phases in the different steels but on the characterisation of the surface oxide layer and its dissolution mechanism using surface spectroscopy and electrochemical techniques e.g. LSV.

\section{XPS analysis of oxide passivation layer}

In order to establish the presence of the oxide layer on the stainless steel surface and to estimate the rate of formation of this layer in an aerobic environment XPS was used as a surface specific technique. Fig. 1 shows a set of X-ray photoelectron data for the $\mathrm{Fe}(2 \mathrm{p}), \operatorname{Cr}(2 \mathrm{p})$ and $\mathrm{O}(1 \mathrm{~s})$ regions of the spectrum. Fig. 1a and b show the $\mathrm{Fe}(2 \mathrm{p})$ and $\mathrm{Cr}(2 \mathrm{p})$ regions, respectively, for a sample of 304 stainless steel that was cleaned in situ with an Ar ion etch. The two line features visible in each spectrum represent the two spin components of the $2 p$ region $(1 / 2$ and $3 / 2$ ). In each case the spectra show that only a single metal

Table 1

\begin{tabular}{|c|c|c|c|c|c|c|c|}
\hline AISI grade & $\% \mathrm{C}_{\max }$ & $\% \mathrm{Si}_{\max }$ & $\% \mathrm{Mn}_{\max }$ & $\% \mathrm{Cr}$ & $\% \mathrm{Mo}_{\max }$ & $\% \mathrm{Ni}$ & Other ${ }_{\text {max. }}$ \\
\hline 410 & 0.15 & 0.50 & 1.00 & $11.5-13.0$ & - & - & - \\
\hline 302 & 0.15 & 1.00 & 2.00 & $17.0-19.0$ & - & $8.0-10.0$ & - \\
\hline 304 & 0.08 & 1.00 & 2.00 & $17.5-20.0$ & - & $8.0-10.5$ & - \\
\hline 316 & 0.08 & 1.00 & 2.00 & $16.0-18.0$ & 2.5 & $10.0-14.0$ & - \\
\hline 347 & 0.08 & 1.00 & 2.00 & $17.0-19.0$ & - & $9.0-13.0$ & $10 \times \mathrm{C} \mathrm{Nb}, \mathrm{Ta}$ \\
\hline
\end{tabular}



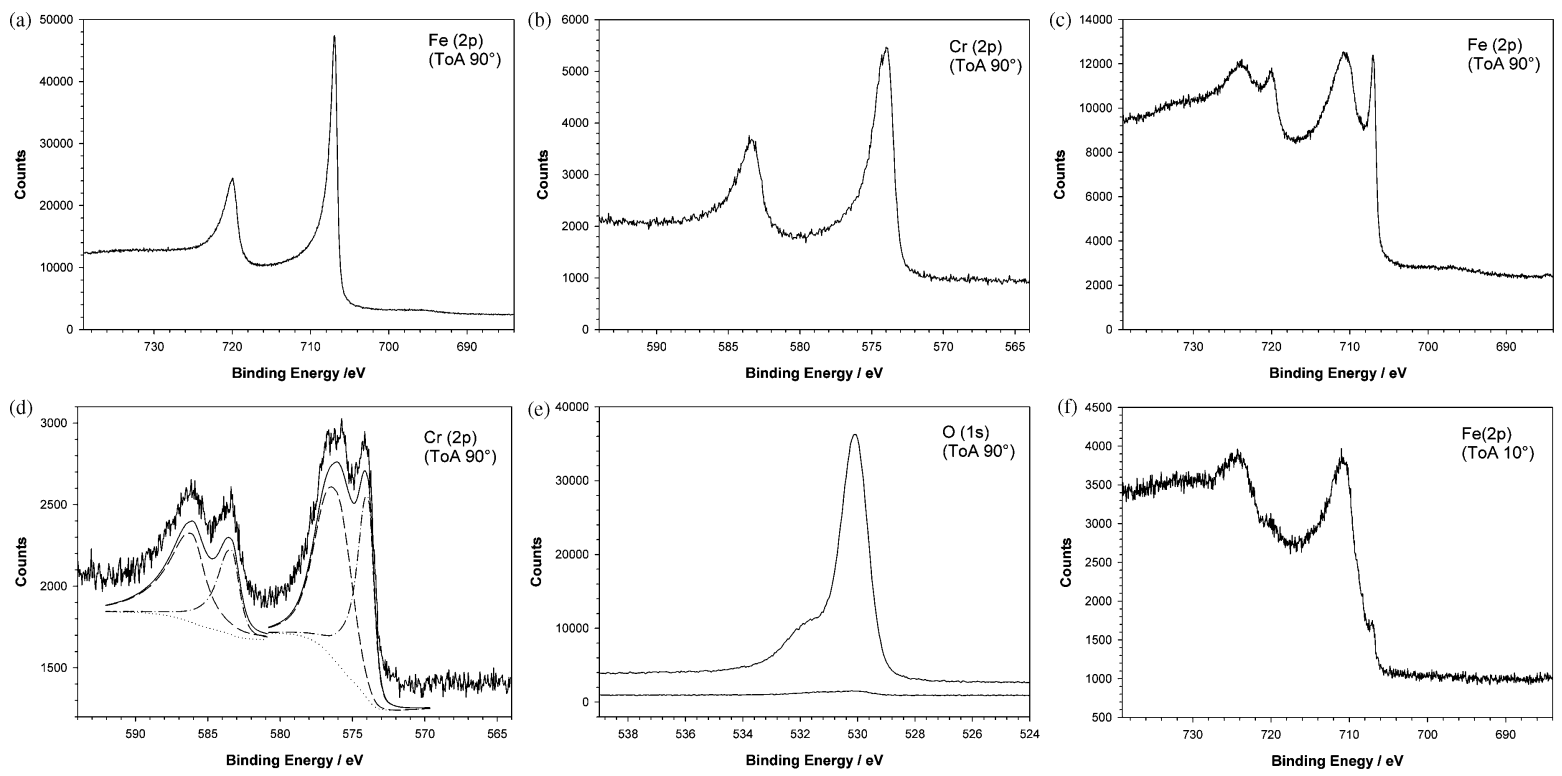

Fig. 1 X-ray photoelectron spectra recorded on a polished piece of stainless steel 304 etched for 2 min with an Ar ion beam in the analysis chamber of the spectrometer; before; (a) $\mathrm{Fe}(2 \mathrm{p}$ ) region, (b) $\mathrm{Cr}(2 \mathrm{p}$ ) region and after (c) $\mathrm{Fe}(2 \mathrm{p}$ ) region, (d) Cr(2p) exposure to air for 10 min. Dotted and dashed lines (d) show the two components of the spectral deconvolution for the $2 p(3 / 2)$ and $2 p(1 / 2)$ lines of the Cr( $2 p$ ) region. (These are offset by 200 counts for clarity.) Oxygen region of the XPS spectrum is shown in (e). Fe( $2 \mathrm{p})$ region recorded after air exposure at a TOA of $10^{\circ}$ is shown in (f).

species is present at the surface and the binding energies are indicative of metallic $\mathrm{Fe}^{0}$ and metallic $\mathrm{Cr}^{0} .{ }^{21}$ The metal sample was then brought out of the analysis chamber of the XPS instrument (where gas pressure is $c a .10^{-9}$ Torr) to rest in air at room temperature for a period of $10 \mathrm{~min}$. The same sample was then returned to the XPS instrument for analysis and comparison. Fig. 1c and d show the XPS spectra of the Fe(2p) and $\operatorname{Cr}(2 p)$ regions, respectively, for the sample exposed to air. Each of these clearly shows two features; the original metallic lines as well as a pair of broader lines shifted to higher binding energy. The binding energy and shape of these features are consistent with the formation of surface $\mathrm{Fe}$ and $\mathrm{Cr}$ oxides. Spectral deconvolution (iterative curve fitting) of both the $2 p(1 / 2)$ and $2 p(3 / 2)$ components of the $\mathrm{Cr}$ and $\mathrm{Fe}$ regions, using the two component environments shown in Fig. 1d, reveals that approximately $70 \%( \pm 10)$ of the surface $\mathrm{Fe}$ is in an oxidised form, while $65 \%( \pm 5)$ of the $\mathrm{Cr}$ is present as a surface oxide. Fig. 1e shows the $\mathrm{O}(1 \mathrm{~s})$ region of the same sample before and after exposure to air. Little detectable $\mathrm{O}$ is present on the cleaned surface whereas the $\mathrm{O}(1 \mathrm{~s})$ signal for the exposed sample is very strong. It is noteworthy, however, that some of the observed $\mathrm{O}(1 \mathrm{~s})$ intensity is probably due to adsorbed $\mathrm{O}$ containing organics. This is evidenced by a small amount of $\mathrm{C}$ also visible (in the $\mathrm{C}(1 \mathrm{~s})$ region) on the surface of the exposed sample.

The surface penetration depth of the XPS experiment is limited to a few nanometres because of inelastic collisions and recapture events. However, the sub surface volume over which the photoelectron response is integrated is a function of the angle that the electron energy analyser makes with the sample surface (take off angle, TOA). The volume of integration is maximised at a TOA of $90^{\circ}$ whereas at smaller values of TOA the technique becomes much more surface specific. In the experiments above the TOA was set to $90^{\circ}$. The experiment was then repeated at a TOA of $10^{\circ}$; this effectively probes only the outer most 1-2 nm of the surface. Fig. 1f shows the Fe(2p) of the exposed sample with a TOA of $10^{\circ}$. Here only the Fe oxide layer is visible with no sign of the underlying metallic iron.

Electrochemistry of stainless steels in EG/ChCl. The results of a series of LSV experiments carried out with various grades of stainless steels in $2 \mathrm{EG}: 1 \mathrm{ChCl}$ mixture are summarised in Fig. 2. In these experiments the steel wire was formed into the working electrode of a 3 electrode cell. This was achieved by encasing the wire in a glass tube using epoxy resin; the wire surface was exposed by polishing. A fresh surface was obtained by manual abrasion using glass paper (see Experimental section). Direct comparison, and interconversion, of potential data from $\mathrm{EG} / \mathrm{ChCl}$ liquids is difficult because of liquid junction effects associated with aqueous (or other solvent) reference electrodes. Consequently a silver wire pseudo reference electrode was used. Whilst the reference potential of the silver wire is, in principle, prone to fluctuation we have observed that the potential is dominated by the chloride ion concentration which is both very high $\left(4.18 \mathrm{~mol} \mathrm{dm}^{-3}\right)$ and essentially invariant throughout our experiments.

Five freshly abraded steel electrodes, having individual compositions summarised in Table 1 were prepared and stored overnight in deionised water. The steel electrodes were then dried and transferred into a fresh mixture of $\mathrm{EG}$ and $\mathrm{ChCl}$ (in a molar ratio $2 \mathrm{EG}: 1 \mathrm{ChCl}$ ). Fig. 2 a shows the $\mathrm{LSV}$ response for each of the different steels. All the sample steels show a single anodic peak at a potential in excess of $1.5 \mathrm{~V}$. The variation in peak current is quite large; this is due to a variation in surface area (electrodes with $0.5 \mathrm{~mm}$ and 

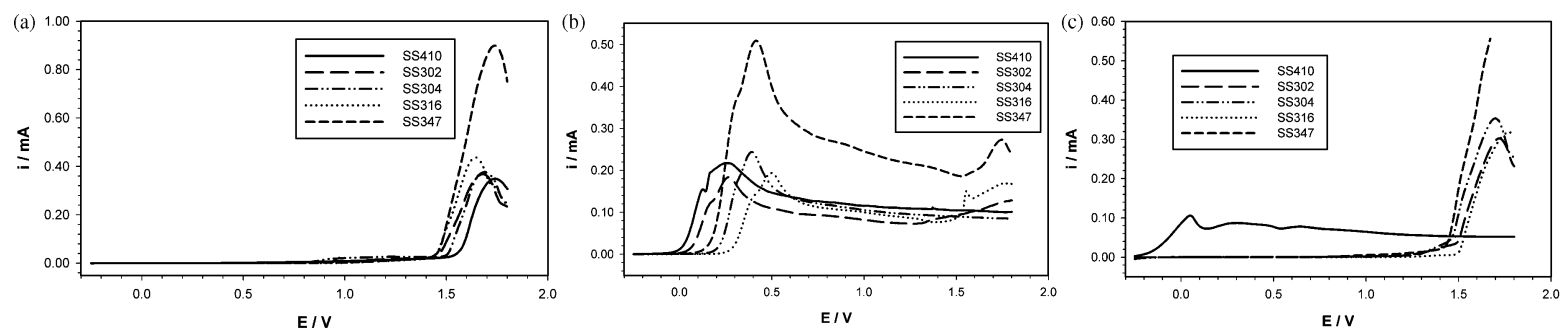

Fig. 2 Linear sweep voltammograms recorded for various grade steel wires ( $0.5 \mathrm{~mm} \mathrm{diam}$. except SS347 $1.0 \mathrm{~mm}$ diam. $)$ in ethylene glycol choline chloride ionic liquid. The voltammograms were recorded at a potential scan rate of $20 \mathrm{mV} \mathrm{s}^{-1}$ using a silver wire pseudo reference electrode: (a) recorded after abrasion and soaking in deionised water; (b) recorded after abrasion, soaking in deionised water with subsequent in situ abrasion in the ionic liquid; (c) recorded after soaking in proprietary chemical pickling solution.

$1.0 \mathrm{~mm}$ diameters were used; see Fig. 2) and also by surface roughness effects resulting from the manner in which the electrodes were prepared. ${ }^{22}$ We interpret the onset of the dissolution peak as representing the breakdown of the oxide film which exposes the underlying metal surface. The most anodic onset potential is exhibited by the SS410 sample and the least anodic by the SS316. The SS316 alloy has the highest Ni content of these samples where as the SS410 alloy has the lowest (with no Ni).

The electrodes were again abraded and stored in deionised water as before. Subsequently they were dried, transferred to a cell containing the ionic liquid and abraded again in situ in an attempt to physically remove the oxide film and to minimise the rate of reoxidation. Fig. $2 b$ shows the LSV responses of the steel electrodes prepared this way without an oxide film. These responses are once again dominated by a single anodic dissolution peak, however, all the dissolution peaks are shifted cathodically by $1100-1800 \mathrm{mV}$. Interestingly, under these conditions, the SS410 sample has the most cathodic dissolution onset potential whilst the SS316 has the most anodic one. Consequentially it follows that for any given steel composition, at an electrode potential in the region lying between the two onset potentials (defined by the LSV curves, Fig. $2 \mathrm{a}$ and $\mathrm{b}$ i.e. in the presence and absence, respectively, of the oxide film), metal dissolution is thermodynamically favourable yet strongly inhibited by the presence of the surface oxide layer. For example at a potential of $+0.75 \mathrm{~V}$, Fig. $2 b$, where little or no oxide film is present, the magnitude of anodic current is in excess of $100 \mu \mathrm{A}$ for all samples whereas at the equivalent potential, Fig. 2a where oxide film is established, the current values are close to zero.

The significance of this is that in 400 series the oxide is thermodynamically the most difficult to remove but the underlying metal is significantly easier to dissolve than any of the 300 series steels. This is important because in practical situations the electropolishing of 400 series steels at low current densities will lead to incomplete oxide removal which will cause pitting dissolution where the underlying alloy is exposed. This is indeed the case, even in aqueous solutions. One practical remedy is to use an acid pickle to chemically remove the oxide film.

In a separate experiment the removal of oxide film from the steel samples by chemical dissolution in a commercial "pickling" solution was explored. The "pickling" solution consisted of a proprietary mix of phosphoric acid and a surfactant. ${ }^{23}$ The steel electrode surfaces were prepared in the same way as those for which the data are shown in Fig. 2a, however, in place of deionised water, the electrodes were soaked in pickling solution before being rinsed and transferred to the $\mathrm{EG} / \mathrm{ChCl}$ liquid. These data are presented in Fig. 2c. Here the linear sweep voltammograms for most of the steels are very similar to those shown in Fig. 2a. Consequently it is clear that the chemical pickle does not remove the oxide layer from most of the steel surfaces. However, the response for the 410 grade sample bears a much stronger resemblance to that in Fig. 2b, indicating that for this steel the pickle is effective at oxide film removal.

\section{SEM and EDX analysis}

We set out to compare the surface morphology and composition of polished and unpolished regions of the steels. This was accomplished by preparing steel samples with regions masked off using an acrylic resin insoluble in the ionic liquid. The samples were then subjected to the electropolishing regime and the mask was subsequently removed with acetone. This methodology facilitates both qualitative and quantitative comparison of composition and morphology over a spatially confined region of the same surface. Fig. 3a shows an optical photograph of one such sample (stainless steel 304) after the removal of the resin mask: here the polished (p) and unpolished (u) regions are clearly visible. This sample was transferred into the SEM and the image presented as Fig. 3b shows the unpolished (masked) surface. The magnitude and type of grain boundary pattern are characteristic of stainless steel sheet. In the image presented as Fig. 3c both polished and unpolished regions are clearly visible, separated by a boundary defined by the mask. The polished region appears very much smoother with no trace of the grain boundary pattern seen in the unpolished region. Since one of the motivations for the commercial electropolishing process is to improve corrosion resistance of the substrate it was important for us to establish that the polishing mechanism in the ionic liquid did not result in gross changes of the elemental composition at the interface i.e. dealloying. Data obtained from energy dispersive X-ray analysis (EDX), using a beam spot on each side of the boundary observed in the image shown in Fig. 3c, are presented in Table 2. These show clearly that the composition of the alloy (insofar as we have analysed for the main components only) is unchanged by the polishing process and therefore that no dealloying occurs. 
(a)

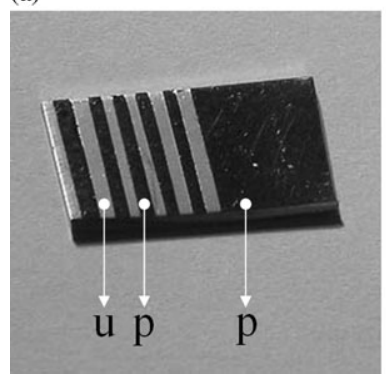

(b)

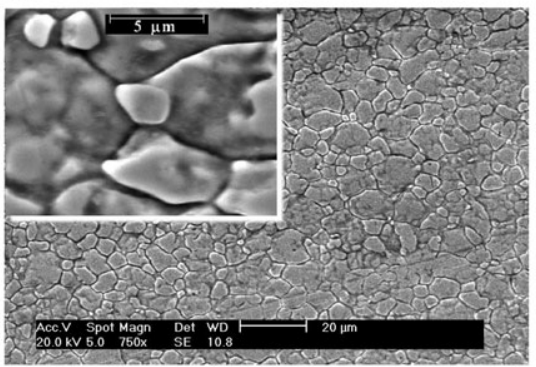

(c)

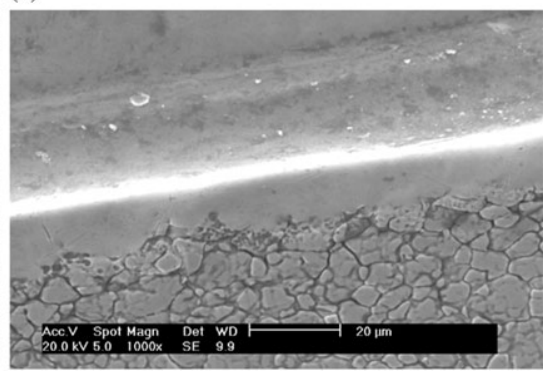

Fig. 3 (a) Photograph of a sample of stainless steel 304 masked (with acrylic resin) and polished with $c a$. 1 mm stripes showing unpolished (u) and polished (p) regions. The mask was removed with acetone (b) Scanning electron micrograph of the unpolished region of the sample shown in (a), high magnification insert. (c) Scanning electron micrograph showing the transition between polished (top) and unpolished (bottom) regions of the sample.

Table 2

\begin{tabular}{llll}
\hline & \multicolumn{3}{l}{ Sample composition (EDX spot analysis) } \\
\cline { 2 - 4 } Sample area & $\% \mathrm{Fe}$ & $\% \mathrm{Cr}$ & $\% \mathrm{Ni}$ \\
\hline Polished & 68.9 & 17.6 & 10.1 \\
Unpolished & 68.8 & 17.3 & 10.2 \\
\hline
\end{tabular}

\section{XPS imaging}

The compositional information obtained from EDX data on a sample is averaged over a volume element defined by the spallation depth of the electron beam. This, in turn, is a function of the experimental beam energy (accelerator voltage). In our experiments the spallation depth of the electron beam was between 1 and $3 \mu \mathrm{m}$. Consequently the EDX data give no information about alloy composition at the solid liquid interface. In order to probe the manifestation and magnitude of any surface dealloying, samples of stainless steels (patterned in the way described above) were examined using an XPS imaging technique. Fig. 4 shows XPS images acquired in the $\mathrm{Cr}(2 \mathrm{p})$ and $\mathrm{Fe}(2 \mathrm{p})$ binding energy regions for the sample whose optical and SEM images are presented in Fig. 3. This technique records a 1-D image of the sample along the direction perpendicular to the direction of the patterned stripes. The $Y$-axis of the image corresponds to binding energy, the $X$-axis defines position on the sample and the colour intensity scale corresponds to electron counts. ${ }^{24,25}$ Both of the XPS images, shown as Fig. $4 \mathrm{a}$ and b, exhibit prominent vertical line features. These occur at the same lateral position on both images and correspond to the delineation of the polished and unpolished regions. The lateral feature (blue in colour) that runs across the image presented in Fig. 4a corresponds to the $\mathrm{Fe}(2 \mathrm{p} 3 / 2)$ spectral line whilst that in Fig. $4 \mathrm{~b}$ corresponds to the $\mathrm{Cr}(2 \mathrm{p} 3 / 2)$ spectral line. There is some variation in the intensity of both the $\mathrm{Fe}(2 \mathrm{p})$ and the $\mathrm{Cr}(2 \mathrm{p})$ signals across the sample. This might suggest some variation in surface composition between the two regions. However there is no obvious discontinuity in the colour across the boundaries. In fact, the observed variation in signal intensity can be accounted for by the variation in detector sensitivity. This is illustrated by Fig. 4c which shows an XPS image (on the Fe (2p 3/2) line) of a homogeneous region of polished steel from the same sample. The same variation of signal intensity is observed here in the absence of surface morphology or compositional contrast. From this qualitative analysis of these data we conclude that there is no evidence of surface dealloying resulting from electropolishing. This is consistent with our solution analyses previously presented. ${ }^{19,20}$

\section{Surface morphology}

In order to study the surface morphology and roughness of the steels before and after polishing and in an attempt to quantify (a)

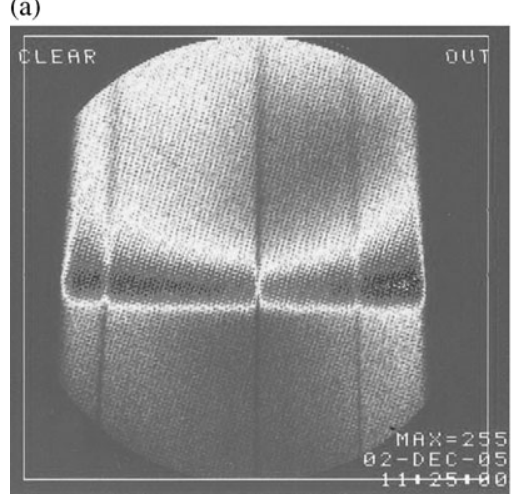

(b)

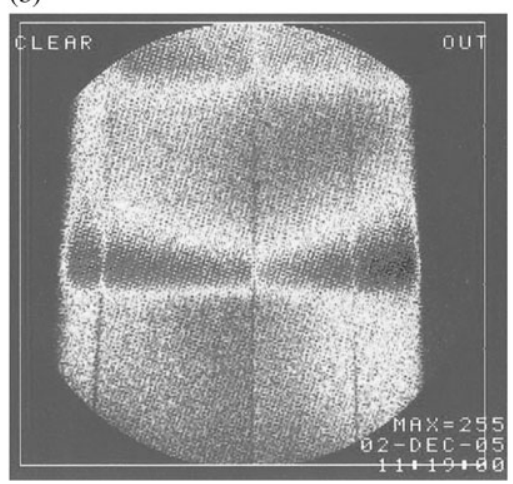

(c)

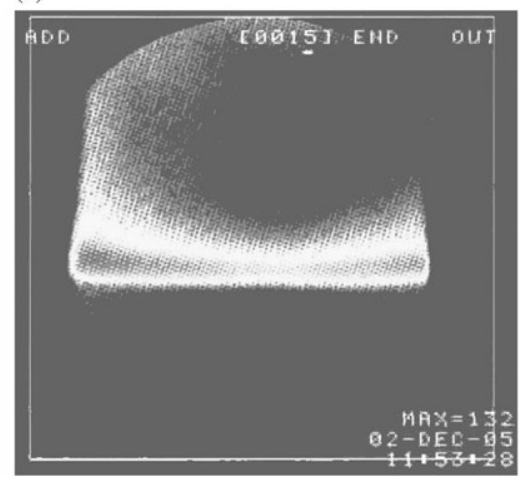

Fig. 4 XPS images of the sample, Fig. 3, (a) and (b) recorded in a direction perpendicular to the patterned stripes in the $\mathrm{Fe}(2 \mathrm{p} \mathrm{3/2)} \mathrm{and} \mathrm{Cr}(2 \mathrm{p} \mathrm{3/2})$ spectral regions, respectively, (c) recorded on a homogeneous polished part of the sample in the $\mathrm{Fe}(2 \mathrm{p} 3 / 2)$ region. Distance traversed along the sample $(c a .4 \mathrm{~mm})$ is recorded on the $x$-axis of the image. 

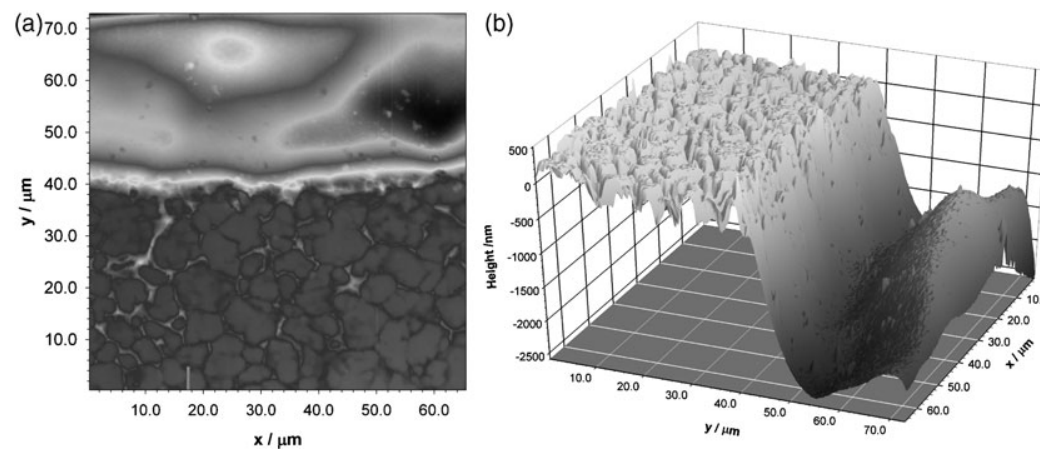

(c)

Fig. 5 Resonant mode (ca. $300 \mathrm{kHz}$ ) AFM images (recorded in air at a scan rate of $0.5 \mathrm{~Hz}, 256$ lines) of the sample, Fig. 3, at the interface between polished and unpolished regions, (a) tip-height contrast projection and (b) 3-D surface map showing the corresponding height scale, (c) slice through the surface at $X=35.6 \mu \mathrm{m}$ showing the tip height data for a single line scan.

the etch rate we examined the surfaces using contact and tapping mode AFM. Fig. 5 shows height contrast images of a sample of stainless steel 304 recorded across the boundary between polished and unpolished areas over a length scale of ca. $74 \times 72 \mu \mathrm{m}$. The height contrast projection, Fig. 5a, shows clearly defined areas of morphology contrast in much the same way as the corresponding SEM image, Fig. 3c. The surface reconstruction using the height data from Fig. 5a, is shown in Fig. 5b. A cross-section of this surface, corresponding to a single line scan at $X=35.6 \mu \mathrm{m}$, is shown in Fig. 5c. The unpolished area of the surface shows the same surface roughness and grain boundary structure as that observed in the SEM. Although the surface is very rough (giving rise to its optically dull finish), with feature sizes of up to $500 \mathrm{~nm}$, it is also very flat; see Fig. $5 \mathrm{c}$ in the region $y<30 \mu \mathrm{m}$. This is no doubt a consequence of the production technique (rolling). In contrast, the polished region, Fig. $5 \mathrm{c}$ in the region $y>40 \mu \mathrm{m}$, has a bright optical finish, it is very smooth but there are large scale fluctuations in height. Surface roughness can be expressed as a peak-trough height, $Z_{\max }$, measured over a designated area of the image. Quantification of the images described above was carried out using a square sample area $\left(25 \mu^{2}\right)$ at random positions across the polished and unpolished regions. Mean values of $Z_{\max }$ (calculated over 10 measurements) for the unpolished and polished surface were determined as $497( \pm 50) \mathrm{nm}$ and $88( \pm 10) \mathrm{nm}$, respectively. The value of $Z_{\max }$ for the unpolished surface is larger than it might be anticipated but this is almost certainly due to surface inclination (i.e. the surface is not flat) rather than intrinsic roughness. An alternative measure of surface roughness can be calculated from the difference ratio of geometric sample area, $A_{G}$, (here this is $25 \mu \mathrm{m}^{2}$ ) to the real surface area, $A_{R}$, calculated from the height data. Surface roughness, $R$, is then expressed as a percentage according to eqn (1).

$$
R=\left(\frac{A_{R}-A_{G}}{A_{G}}\right) \times 100 \%
$$

By this method the unpolished region of the sample has an average roughness of $7.94 \%$ whereas the polished region has a value of just $0.15 \%$. At the edge of the boundary between polished and unpolished areas the extent of metal etch is visible. Up to $2 \mu \mathrm{m}$ of metal is lost at the boundary; this falls to $1 \mu \mathrm{m}$ at distances further away from the mask edge. There is no evidence of undercutting at the mask suggesting that the etch is isotropic.

\section{Liquid AFM in situ studies}

In an attempt to understand better the electrodissolution process we have studied the surface transformation of the steel during polishing using in situ liquid AFM. This was accomplished with a liquid interface attachment for the AFM instrument together with the corresponding probes. Although the liquid interface attachment was designed for use in an aqueous environment we have found that it also functions well in the ionic liquid. The attachment was operated in tapping (resonant) mode and despite the relatively high viscosity of the liquid (50 mPa s @293 K) a tip resonance was easily located at between $6-8 \mathrm{kHz}$ without the necessity for an excessively high drive amplitude. A schematic representation of the electrochemical cell used for the in situ AFM experiment is shown in Fig. 6. The cell was constructed using a SS316 anode plate for the base and a copper foil cathode. The copper foil was insulated from the anode with adhesive PVC tape and a rubber $O$-ring was fixed over both electrodes (using silicone rubber adhesive) as containment for the liquid electrolyte. To minimise the influence of the geometry of the probe assembly on the current density near the surface the steel plate was imaged through a $5 \mathrm{~mm}$ hole in the $\mathrm{Cu}$ cathode. Real time imaging of the process is experimentally very difficult because small fragments and particles of metal are discharged from the surface during the dissolution process. These diffuse through the liquid passing between the laser source, the tip assembly and the photodetector. The resultant scattering of the laser light results in perturbation (noise) and ultimately loss of feedback control. As a result the images were recorded at discrete intervals before polishing, part way through and at the end of the polishing process. These images are shown in Fig. 7. In order to ensure that the same representative area was imaged each time the tip was not fully withdrawn from the surface (enabling reasonably accurate repositioning) between measurements.

The image of the unpolished steel surface is shown in Fig. 7a. Here the morphology and magnitude of surface roughness are consistent with those observed previously. The diagonal score marks at the bottom left hand corner of this image are 


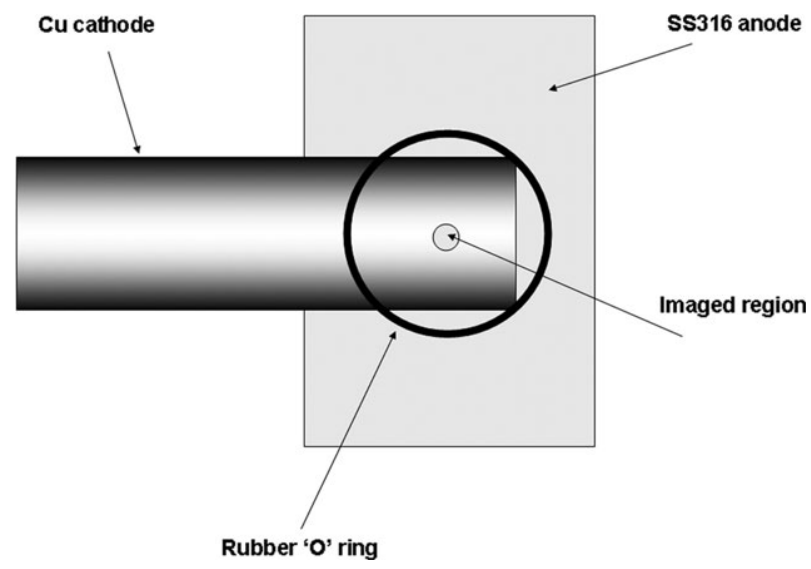

Fig. 6 Schematic diagram of the liquid cell for in situ AFM measurements. The imaged region of the steel was at the center of a hole in the copper cathode $c a .5 \mathrm{~mm}$ in diameter.

rolling marks from the manufacturing process. The resolution of the grain boundary features is good suggesting that there are no tip control problems or imaging artefacts associated with surface wetting. The steel was then subjected to the polishing conditions for 10 min (constant current density@, $25 \mathrm{~mA} \mathrm{~cm}^{-2}$ ). The tip was taken off the surface and the surface debris was removed by circulation of the polishing liquid without moving the probe. The scanning tip was then reengaged and the image presented in Fig. 7c was recorded. This procedure was repeated, the final image after polishing for $25 \mathrm{~min}$ is presented in Fig. 7e. Alongside each of these images is presented height data for a single line scan across the middle of the sample, Fig. 7b, d and f. To facilitate comparison the height scales are identical for each plot although there is no correlation between the absolute values. ${ }^{26}$ These data show
Table 3

\begin{tabular}{lll}
\hline Sample surface & $\begin{array}{l}\text { Surface roughness, } R, \\
\text { (area difference ratio) } / \%\end{array}$ & $Z_{\max } / \mathrm{nm}$ \\
\hline Unpolished & 12.3 & 2390 \\
10 min. polish & 3.9 & 2360 \\
25 min. polish & 1.9 & 2510 \\
\hline
\end{tabular}

clearly the qualitative transition from the unpolished to polished surfaces. The intermediate finish is evident in Fig. 7c showing what remains of the surface features of the unpolished surface. The surface has been eroded first at the most prominent features where the electric field and presumably, current density, are at their greatest. The single line scan data show that although the polished surface is very smooth there are large scale variations in height. These are comparable in size to the unpolished surface but over a much smaller length scale in the latter. Quantitative analysis of the images using the two methods described in the previous section are presented in Table 3. The surface roughness (area difference ratio) shows a marked and progressive downward trend whilst the value of $Z_{\max }$ is largely unchanged. It is noteworthy that the values of $Z_{\text {max }}$ presented in the table do not correspond to the line scans in Fig. 7 because they are measured over the whole image.

\section{Conclusions}

Here we have studied the anodic dissolution process of a number of stainless steel alloys by electrochemical methods and a variety of surface analysis techniques. We conclude from XPS measurements that surface oxide formation at the clean steel surface is rapid under aerobic conditions and from LSV data we have observed that breakdown of this oxide coating is (a)
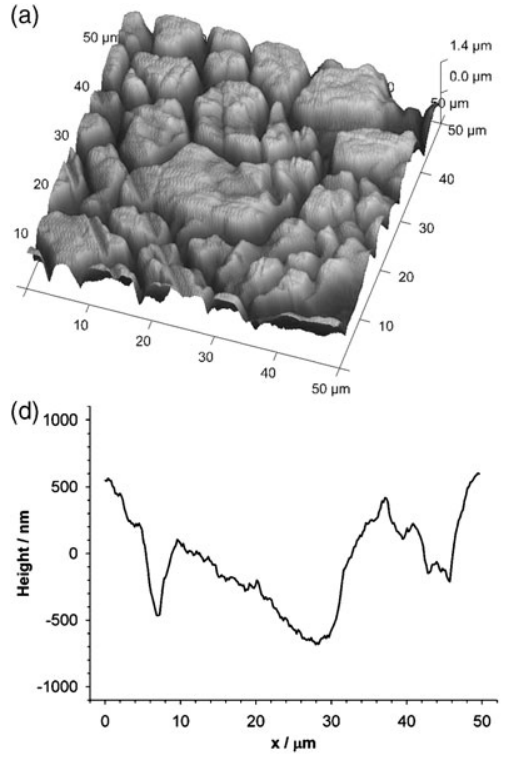

(b)

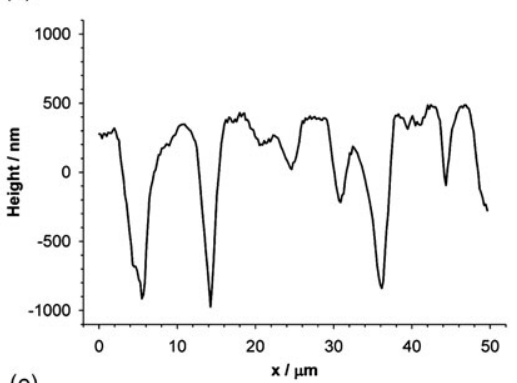

(e)

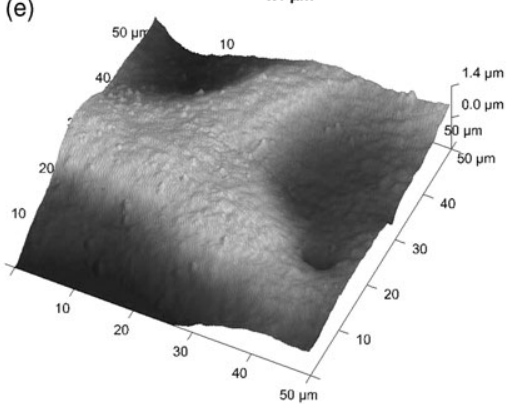

(c)
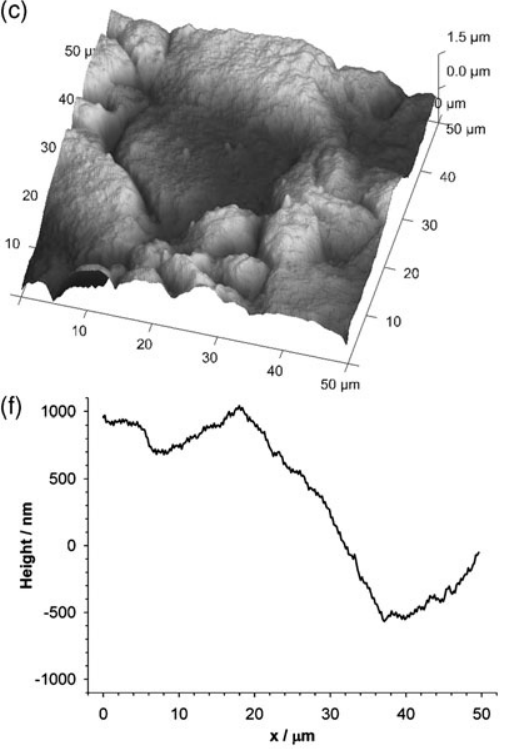

Fig. 7 AFM images, with single line traces alongside, of a stainless steel 316 plate (liquid cell, Fig. 6) recorded in resonant mode (at a frequency of approximately $8 \mathrm{kHz}$ using a scan rate of $1 \mathrm{~Hz}$ and 256 lines) in ionic liquid (a), (b) before polishing; (c), (d); after $10 \mathrm{~min}$ and (e), (f) after 25 min. Constant current density was maintained during polishing at $25 \mathrm{~mA} \mathrm{~cm}^{-2}$. The single line traces were taken laterally (left to right) across the centers of the images shown in (a), (c) and (e). 
the rate limiting factor in the polishing process. Anodic dissolution of metal ions from steel surfaces where the oxide layer was removed in situ was seen to be much faster than at the equivalent alloy surface where oxide coating was encouraged and allowed to remain. Aggressive proprietary chemical treatments of the steels were successful in removing the oxide from only one of the samples studied here (SS410). A combination of XPS imaging and EDX analysis was used to probe the elemental composition of both the outermost surface of the steels as well as the sub-surface volume. Analysis of these data shows that there is no evidence of dealloying of the steels during polishing in this ionic liquid.

Probe microscopy studies in combination with SEM have given both a qualitative and a quantitative insight into the polishing mechanism. Initial etching occurs at the most prominent of the surface features and proceeds until these features have been completely removed. Interestingly the unpolished surface was much flatter, although rougher, than the resultant polished finish. Conversely the polished surface was seen to be very smooth but with similar height variations over a larger distance scale. The size of the crevices and valleys observed in the unpolished surface in relation to the wavelength range of visible light gives rise to its dull finish, whereas the relative smoothness of the polished surface over the 1-2 $\mu \mathrm{m}$ scale results in an optically bright finish. Consequently the larger scale undulations are not detrimental to the subjective quality of the polished surface. With the use of masking techniques we have been able to estimate the extent of etch. During a $5 \mathrm{~min}$ etching process $1 \mu \mathrm{m}$ of metal was removed.

\section{Acknowledgements}

The authors would like to thank the DTI (ETREAT) and the EU (Framework 6 project IONMET, http://www.ionmet.org/) for funding. The authors also wish to thank the EPSRC CCLRC for access to the NCESS facility (Daresbury Laboratory) and Dr Graham Beamson (Daresbury Laboratory) for guidance and helpful discussion.

\section{References}

1 H. Figous and P. A. Jacquet, French Patent No: 707526, 1930.
2 L. E. A. Berlouis and D. J. Schiffrin, Trans. Inst. Met. Finish., 1985, 63, 52.

3 I. L. Alanis and D. J. Schiffrin, Electrochim. Acta, 1979, 27, 837.

4 O. Pietrowski, C. Madore and D. Landolt, J. Electrochem. Soc., 1998, 145, 2362.

5 S. Mohan, D. Kanagaraj, R. Sindhuja, S. Vijayalakshmi and N. G. Renganathan, Trans. Inst. Met. Finish., 2001, 79, 140.

6 D. Landolt, Electrochim. Acta, 1987, 32, 1.

7 P. Wasserscheid and T. Welton, Ionic Liquids in Synthesis, WileyVCH Verlag, Weinheim, Germany, 2003.

8 P. Wasserscheid and W. Keim, Angew. Chem., Int. Ed., 2000, 39, 3772.

9 F. Endres, ChemPhysChem, 2002, 3, 144.

10 Electrochemical Aspects of Ionic Liquids, ed. H. Ohno, John Wiley \& Sons, New York, 2005.

11 A. P. Abbott, G. Capper, D. L. Davies, H. L. Munro, R. K. Rasheed and V. Tambyrajah, Chem. Commun., 2001, 2010.

12 A. P. Abbott, G. Capper, D. L. Davies and R. K. Rasheed, Inorg. Chem., 2004, 43, 3447.

13 A. P. Abbott, G. Capper, D. L. Davies, H. L. Munro, R. K. Rasheed and V. Tambyrajah, Ionic Liquids as Green Solvents Progress and Prospects, ACS Symposium Series, ed. R. D. Rogers and K. R. Seddon, 2003, p. 439.

14 A. P. Abbott, G. Capper, D. L. Davies, R. K. Rasheed and V. Tambyrajah, Trans. Inst. Met. Finish., 2001, 79, 204.

15 A. P. Abbott, G. Capper, D. L. Davies and R. K. Rasheed, Chem.-Eur. J., 2004, 10, 3769.

16 A. P. Abbott, G. Capper, D. L. Davies, R. K. Rasheed, J. Archer and C. John, Trans. Inst. Met. Finish., 2004, 82, 14.

17 A. P. Abbott, G. Capper, D. L. Davies, R. K. Rasheed and V. Tambyrajah, Chem. Commun., 2003, 70.

18 A. P. Abbott, D. Boothby, G. Capper, D. L. Davies and R. K. Rasheed, J. Am. Chem. Soc., 2004, 126, 9142.

19 A. P. Abbott, G. Capper, B. G. Swain and D. A. Wheeler, Trans. Inst. Met. Finish., 2005, 83, 51.

20 A. P. Abbott, G. Capper, K. J. McKenzie and K. S. Ryder, Electrochim. Acta, 2006, 51, 4420.

21 NIST X-ray Photoelectron Spectra Database (version 3.2) at http://www.nist.gov/srd/nist20.htm.

22 This argument is supported by quantification of AFM image data which shows that the ratio of morphological surface area to projected geometric area is often as high as $140 \%$.

23 Anopol Ltd (http://www.anopol.co.uk/) proprietary pickling solution.

24 S. D. Evans, T. M. Flynn, A. Ulman and G. Beamson, Surf. Interface Anal., 1996, 24, 187.

25 U. Vohrer, C. Blomfield, S. Page and A. Roberts, Appl. Surf. Sci., 2005, 252, 61-65.

26 There is no correlation between absolute height values in the figures because the tip was disengaged between image acquisitions. The zero point height reference is an arbitrary function of the engage procedure and dependent on the position of the piezoelectric height motor. 